\title{
CALOGERO-MOSER SPACE, RESTRICTED RATIONAL CHEREDNIK ALGEBRAS AND TWO-SIDED CELLS.
}

\author{
I.G. Gordon And M. Martino
}

\begin{abstract}
We conjecture that the "nilpotent points" of Calogero-Moser space for reflection groups are parametrised naturally by the two-sided cells of the group with unequal parameters. The nilpotent points correspond to blocks of restricted Cherednik algebras and we describe these blocks in the case $G=\mu_{\ell} \prec \mathfrak{S}_{n}$ and show that in type $B$ our description produces an existing conjectural description of two-sided cells.
\end{abstract}

\section{Introduction}

1.1. Smooth points are all alike; every singular point is singular in its own way. Calogero-Moser space associated to the symmetric group has remarkable applications in a broad range of topics; in [3], Etingof and Ginzburg introduced a generalisation associated to any complex reflection group which has also found a variety of uses. The Calogero-Moser spaces associated to a complex reflection group, however, exhibit new behaviour: they are often singular. The nature of these singularities remains a mystery, but their existence has been used to solve the problem of the existence of crepant resolutions of symplectic quotient singularities. The generalised Calogero-Moser spaces are moduli spaces of representations of rational Cherednik algebras and so their geometry reflects the representation theory of these algebras: smooth points correspond to irreducible representations of maximal dimension; singular points to smaller, more interesting representations. In this note we conjecture a strong link between the representations corresponding to some particularly interesting "nilpotent points" of Calogero-Moser space and Kazhdan-Lusztig cell theory for Hecke algebras with unequal parameters. To justify the conjecture we give a combinatorial parametrisation of these points, thus answering a question of [6], and then relate this parametrisation to the conjectures of [1] on the cell theory for Weyl groups of type $B$.

1.2. Let $W$ be a complex reflection group and $\mathfrak{h}$ its reflection representation over $\mathbb{C}$. Let $\mathcal{S}$ denote the set of complex reflections in $W$. Let $\omega$ be the canonical symplectic form on $V=\mathfrak{h} \oplus \mathfrak{h}^{*}$. For $s \in \mathcal{S}$, let $\omega_{s}$ be the skew-symmetric form that coincides with $\omega$ on $\operatorname{im}\left(\operatorname{id}_{V}-s\right)$ and has $\operatorname{ker}\left(\operatorname{id}_{V}-s\right)$ as its radical. Let $\mathbf{c}: \mathcal{S} \longrightarrow \mathbb{C}$ be a $W$ invariant function sending $s$ to $c_{s}$. The rational Cherednik algebra at parameter $t=0$ (depending on c) is the quotient of the skew group algebra of the tensor algebra on $V, T V * W$, by the relations

$$
[x, y]=\sum_{s \in \mathcal{S}} c_{s} \omega_{s}(x, y) s
$$

Received by the editors March 19, 2007. 
for all $x, y \in V$. This algebra is denoted by $H_{\mathbf{c}}$.

Let $Z_{\mathbf{c}}$ denote the centre of $H_{\mathbf{c}}$ and set $A=\mathbb{C}\left[\mathfrak{h}^{*}\right]^{W} \otimes \mathbb{C}[\mathfrak{h}]^{W}$. Thanks to [3, Proposition 4.15] $A \subset Z_{\mathbf{c}}$ for any parameter $\mathbf{c}$ and $Z_{\mathbf{c}}$ is a free $A$-module of rank $|W|$. Let $X_{\mathbf{c}}$ denote the spectrum of $Z_{\mathbf{c}}$ : this is called the Calogero-Moser space associated to $W$. Corresponding to the inclusion $A \subset Z_{\mathbf{c}}$ there is a finite surjective morphism $\Upsilon_{\mathbf{c}}: X_{\mathbf{c}} \longrightarrow \mathfrak{h}^{*} / W \times \mathfrak{h} / W$.

Let $\mathfrak{m}$ be the homogeneous maximal ideal of $A$. The restricted rational Cherednik algebra is $\bar{H}_{\mathbf{c}}=H_{\mathbf{c}} / \mathfrak{m} H_{\mathbf{c}}$. By $\left[3\right.$, PBW theorem 1.3] it has dimension $|W|^{3}$ over $\mathbb{C}$. General theory asserts that the blocks of $\bar{H}_{\mathbf{c}}$ are labelled by the closed points of the scheme-theoretic fibre $\Upsilon^{*}(0)$. We call these points the nilpotent points of $X_{\mathbf{c}}$. By [6, 5.4] there is a surjective mapping

$$
\Theta_{\mathbf{c}}: \operatorname{Irr} W \longrightarrow\left\{\text { closed points of } \Upsilon_{\mathbf{c}}^{*}(0)\right\}=\left\{\text { blocks of } \bar{H}_{\mathbf{c}}\right\},
$$

constructed by associating to any $\boldsymbol{\lambda} \in \operatorname{Irr} W$ an indecomposable $\bar{H}_{\mathbf{c}}$-module, the baby Verma module $M_{\mathbf{c}}(\boldsymbol{\lambda})$. The fibres of $\Theta_{\mathbf{c}}$ partition Irr $W$. We will call this the $C M_{\mathbf{c}^{-}}$ partition.

1.3. Let $W$ be a Weyl group. Let $\mathbf{L}: W \rightarrow \mathbb{Q}$ be a weight function (in the sense of $[1$, Section 2]). Let $\mathcal{H}$ be the corresponding Iwahori-Hecke algebra at unequal parameters, an algebra over the group algebra of $\mathbb{Q}, A=\oplus_{q \in \mathbb{Q}} \mathbb{Z} v^{q}$, which has a basis $T_{w}$ for $w \in W$, with multiplication given by the rule

$$
T_{s} T_{w}= \begin{cases}T_{s w} & \text { if } l(s w)=l(w)+1 \\ T_{s w}+\left(v^{\mathbf{L}(s)}-v^{-\mathbf{L}(s)}\right) T_{w} & \text { if } l(s w)=l(w)-1\end{cases}
$$

where $s \in \mathcal{S}$ and $w \in W$. There is an associated partition of $W$ into two-sided cells, see [9, Chapter 8]. We call these the $K L_{\mathbf{L}}$-cells.

Conjecture. Let $W$ be a Weyl group and let $\mathbf{L}$ be the weight function generated by $\mathbf{L}(s)=c_{s}$ for each $s \in \mathcal{S}$.

(1) There is a natural identification of the $C M_{\mathbf{c}}$-partition and the $K L_{\mathbf{L}}$-partition; this is induced by attaching a $K L_{\mathbf{L}}$-cell to an irreducible $W$-representation via the asymptotic algebra $J,[9,20.2]$.

(2) Let $\mathcal{F}$ be a $K L_{\mathbf{L}}$-cell of $W$ and let $M_{\mathcal{F}}$ be the closed point of $\Upsilon_{\mathbf{c}}^{*}(0)$ corresponding to $\mathcal{F}$ by $(1)$. Then $\operatorname{dim}_{\mathbb{C}}\left(\Upsilon_{\mathbf{c}}^{*}(0)_{M_{\mathcal{F}}}\right)=|\mathcal{F}|$.

The existence of the asymptotic algebra mentioned in (1) is still a conjecture, depending on Lusztig's conjectures P1-P15 in [9, Conjecture 14.2].

1.4. This conjecture generalises the known results about the blocks of $\bar{H}_{\mathbf{c}}$ and about the fibre $\Upsilon_{\mathbf{c}}^{*}(0)$.

- [6, Corollary 5.8] If $X_{\mathbf{c}}$ is smooth then $\Theta_{\mathbf{c}}$ is bijective, making the $C M_{\mathbf{c}^{-}}$ partition trivial. If $S \in \operatorname{Irr} W$ then $\operatorname{dim}_{\mathbb{C}}\left(\Upsilon_{\mathbf{c}}^{*}(0)_{M_{\mathcal{S}}}\right)=\operatorname{dim}_{\mathbb{C}}(S)^{2}$.

- $\Theta_{\mathbf{c}}$ is not bijective when $W$ is a finite Coxeter group of type $D_{2 n}, E, F, H$ or $I_{2}(m)(m \geq 5),[6$, Proposition 7.3]. In all of these cases there are non-trivial two-sided cells.

- Both the $a$-function and the $c$-function are constant across fibres of $\Theta_{\mathbf{c}},[7$, Lemma 5.3 and Proposition 9.2]. This should be a property of two-sided cells. 
1.5. In Theorem 2.5 we will give a combinatorial description of the $C M_{\mathbf{c}}$-partition when $W=G(\ell, 1, n)=\mu_{\ell} \prec \mathfrak{S}_{n}$, and then in Theorem 3.3 we will provide evidence for the conjecture by showing that the $C M_{\mathbf{c}}$-partition agrees with the conjectural description of the $K L_{\mathbf{L}}$-partition for $W=G(2,1, n)$, the Weyl group of type $B_{n}$, given in [1, Section 4.2].

1.6. An advantage of the Cherednik algebras is that the $C M_{\mathbf{c}}$-partition exists for any complex reflection group whereas, at the moment, a cell theory only exists for Coxeter groups. However, following [12], one can introduce Rouquier families as a sensible extension of the notion of the $K L_{\mathbf{L}}$-partition to nearly all complex reflection groups and any parameters. In [10] counterparts to the above conjecture are proposed for complex reflection groups in terms of families, and supporting evidence is gathered.

\section{Blocks for $W=G(\ell, 1, n)$}

2.1. Let $\ell$ and $n$ be positive integers. Let $\mu_{\ell}$ be the group of $\ell$-th roots of unity in $\mathbb{C}$ with generator $\gamma$ and let $\mathfrak{S}_{n}$ be the symmetric group on $n$ letters. Let $W$ be the wreath product $G(\ell, 1, n)=\mu_{\ell} \prec \mathfrak{S}_{n}=\left(\mu_{\ell}\right)^{n} \rtimes \mathfrak{S}_{n}$ acting naturally on $\mathfrak{h}=\mathbb{C}^{n}$.

2.2. Let $\mathcal{P}(n)$ denote the set of partitions of $n$ and $\mathcal{P}(\ell, n)$ the set of $\ell$-multipartitions of $n$. The set Irr $W$ can be identified naturally with $\mathcal{P}(\ell, n)$ so that the trivial representation corresponds to $((n), \emptyset, \ldots, \emptyset)$, e.g. [8, Theorem 4.4.3]. Given an element $\mathbf{s} \in \mathbb{Z}_{0}^{\ell}=\left\{\left(s_{1}, \ldots, s_{\ell}\right) \in \mathbb{Z}^{\ell}: s_{1}+\cdots+s_{\ell}=0\right\}$ there is an associated $\ell$-core (a partition from which no $\ell$-hooks can be removed). The inverse of the process assigning to a partition its $\ell$-core and $\ell$-quotient defines a bijection

$$
\mathbb{Z}_{0}^{\ell} \times \coprod_{n} \mathcal{P}(\ell, n) \longrightarrow \coprod_{n} \mathcal{P}(n), \quad(\mathbf{s}, \boldsymbol{\lambda}) \mapsto \tau_{\mathbf{s}}(\boldsymbol{\lambda}) .
$$

A detailed discussion of this can be found in [8, Section 2.7] or [7, Section 6].

2.3. The Young diagram of a partition $\lambda$ will always be justified to the northwest (one of the authors is English); we will label the box in the $p$ th row and $q$ th column of $\lambda$ by $s_{p q}$. With this convention the residue of $s_{p q}$ is defined to be congruence class of $p-q$ modulo $\ell$. Recall that $s_{p q}$ is said to be $j$-removable for some $0 \leq j \leq \ell-1$ if it has residue $j$ and if $\lambda \backslash\left\{s_{p q}\right\}$ is the Young diagram of another partition, a predecessor of $\lambda$. We say that $s_{p q}$ is $j$-addable to $\lambda \backslash\left\{s_{p q}\right\}$.

Let $J \subseteq\{0, \ldots, \ell-1\}$. We define the $J$-heart of $\lambda$ to be the sub-partition of $\lambda$ which is obtained by removing as often as possible $j$-removable boxes with $j \in J$ from $\lambda$ and its predecessors. A subset of $\mathcal{P}(n)$ whose elements are the partitions with a given $J$-heart is called a $J$-class.

2.4. We will use the "stability parameters" of [7] $\boldsymbol{\theta}(\mathbf{c})=\left(\theta_{0}, \ldots, \theta_{\ell-1}\right)$ defined by $\theta_{k}=-\delta_{0 k} c_{(i, j)}+\sum_{t=1}^{\ell-1} \eta^{t k} c_{\gamma^{t}}$ for $0 \leq k \leq \ell-1, \eta$ a primitive $l$-th root of unity and an arbitrary transposition $(i, j) \in \mathfrak{S}_{n}$ : they contain the same information as c. Following [7, Theorem 4.1] we set $\Theta=\left\{\left(\theta_{0}, \ldots, \theta_{\ell-1}\right) \in \mathbb{Q}^{\ell}\right\}$ and $\Theta_{1}=\{\boldsymbol{\theta} \in \Theta$ : $\left.\theta_{0}+\cdots+\theta_{\ell-1}=1\right\}$.

Let $\tilde{\mathfrak{S}}_{\ell}$ denote the affine symmetric group with generators $\left\{\sigma_{0}, \ldots, \sigma_{\ell-1}\right\}$. It acts naturally on $\Theta$ by $\sigma_{j} \cdot\left(\theta_{0}, \ldots, \theta_{\ell-1}\right)=\left(\theta_{0}, \ldots, \theta_{j-1}+\theta_{j},-\theta_{j}, \theta_{j}+\theta_{j+1}, \ldots, \theta_{\ell-1}\right)$. This restricts to the affine reflection representation on $\Theta_{1}$ : the walls of $\Theta_{1}$ are the 
reflecting hyperplanes and the alcoves of $\Theta_{1}$ are the connected components of (the real extension of) $\Theta_{1} \backslash$ wwalls $\}$. Let $A_{0}$ be the alcove containing the point $\ell^{-1}(1, \ldots, 1)$ : its closure is a fundamental domain for the action of $\tilde{\mathfrak{S}}_{\ell}$ on $\Theta_{1}$. The stabiliser of a point $\boldsymbol{\theta} \in \bar{A}_{0}$ is a standard parabolic subgroup of $\tilde{\mathfrak{S}}_{\ell}$ generated by simple reflections $\left\{\sigma_{j}: j \in J\right\}$ for some subset $J \subseteq\{0, \ldots, \ell-1\}$. We call this subset the type of $\boldsymbol{\theta}$. The type of an arbitrary point $\boldsymbol{\theta} \in \Theta_{1}$ is defined to be the type of its conjugate in $\bar{A}_{0}$.

2.5. We have an isomorphism $\tilde{\mathfrak{S}}_{\ell} \cong \mathbb{Z}_{0}^{\ell} \rtimes \mathfrak{S}_{l}$ with $\mathfrak{S}_{\ell}=\left\langle\sigma_{1}, \ldots, \sigma_{\ell-1}\right\rangle$ and the elements of $\mathbb{Z}_{0}^{\ell}$ corresponding to translations by elements of the dual root lattice $\mathbb{Z} R^{\vee}$. The symmetric group $\mathfrak{S}_{\ell}$ acts on $\mathcal{P}(\ell, n)$ by permuting the partitions comprising an $\ell$-multipartition.

Theorem. Assume that $\boldsymbol{\theta}(\mathbf{c}) \in \Theta_{1}$, so that $\boldsymbol{\theta}(\mathbf{c})$ has type $J$ and belongs to $(\mathbf{s}, w) \cdot \bar{A}_{0}$ for some $(\mathbf{s}, w) \in \tilde{\mathfrak{S}}_{\ell}$. Then $\boldsymbol{\lambda}, \boldsymbol{\mu} \in \operatorname{Irr} W=\mathcal{P}(\ell, n)$ belong to the same block of $\bar{H}_{\mathbf{c}}$ if and only if $\tau_{\mathbf{s}}(w \cdot \boldsymbol{\lambda})$ and $\tau_{\mathbf{s}}(w \cdot \boldsymbol{\mu})$ belong to the same $J$-class. In other words, the $C M_{\mathbf{c}}$-partition is governed by the $J$-classes.

Proof. Rescaling gives an isomorphism between $\bar{H}_{\mathbf{c}}$ and $\bar{H}_{\mathbf{c} / 2}$ so we can replace $\mathbf{c}$ by c/2. By $[6,5.4]$ we must show that the baby Verma modules $M_{\mathbf{c} / 2}(\boldsymbol{\lambda})$ and $M_{\mathbf{c} / 2}(\boldsymbol{\mu})$ give rise to the same closed point of $\Upsilon_{\mathbf{c} / 2}^{*}(0)$ if and only if $\tau_{\mathbf{s}}(w \cdot \boldsymbol{\lambda})$ and $\tau_{\mathbf{s}}(w \cdot \boldsymbol{\mu})$ have the same $J$-class. But the closed points of $\Upsilon_{\mathbf{c} / 2}^{*}(0)$ correspond to the $\mathbb{C}^{*}$-fixed points of $X_{\mathbf{c} / 2}$ under the action induced from the grading on $H_{\mathbf{c} / 2}$ which assigns degree 1 , respectively -1 and 0 , to non-zero elements of $\mathfrak{h}$, respectively $\mathfrak{h}^{*}$ and $W$. By $\left[7\right.$, Theorem 3.10] these agree with the $\mathbb{C}^{*}$-fixed points on the affine quiver variety $\mathcal{X}_{\boldsymbol{\theta}(\mathbf{c})}(n)$ and hence, thanks to $\left[7\right.$, Equation (3)] to the $\mathbb{C}^{*}$-fixed points on the Nakajima quiver variety $\mathcal{M}_{\boldsymbol{\theta}(\mathbf{c})}(n)$. Now the result follows since the combinatorial description of these fixed points in [7, Proposition 8.3(i)] is exactly the one in the statement of the theorem.

2.6. Remarks. (1) The assumption $\boldsymbol{\theta}(\mathbf{c}) \in \Theta_{1}$ imposes two restrictions. First it places a rationality condition on the entries of $\mathbf{c}$; guided by corresponding results for Hecke algebras, [2, Theorem 1.1], we hope that this is not really a serious restriction. Second it forces $c_{(i, j)} \neq 0$; if $c_{(i, j)} \neq 0$ then we can rescale to produce an isomorphism $H_{\mathbf{c}} \cong H_{\lambda \mathbf{c}}$ and hence ensure $\theta_{0}+\cdots+\theta_{\ell-1}=1$.

(2) A generic choice of $\boldsymbol{\theta}(\mathbf{c}) \in \Theta_{1}$ will have type $J=\emptyset$. The corresonding $C M_{\mathbf{c}^{-}}$ partition will then be trivial and thus $X_{\mathbf{c}}$ will be smooth, [3, Corollary 1.14(i)].

\section{The case $W=G(2,1, n)$}

3.1. We now focus on the situation where $W=G(2,1, n)$, the Weyl group of type $B_{n}$. Here there are two conjugacy classes of reflections $s$ and $t$, those associated to $(i, j)$ and $\gamma$ respectively. We will always assume that $\mathbf{c}=\left(c_{s}, c_{t}\right) \in \mathbb{Q}^{2}$ has the property that $c_{s}, c_{t} \neq 0$. Corresponding to the two group homomorphisms $\epsilon_{1}, \epsilon_{2}$ : $W \rightarrow \mathbb{C}^{*}, \epsilon_{k}(i, j)=(-1)^{k}$ for all $(i, j) \in s$ and $\epsilon_{k}(\sigma)=(-1)^{k+1}$, there exist algebra isomorphisms $H_{\left(c_{s}, c_{t}\right)} \cong H_{\left(-c_{s}, c_{t}\right)}$ and $H_{\left(c_{s}, c_{t}\right)} \cong H_{\left(c_{s},-c_{t}\right)}$, [5, 5.4.1]. So, without loss of generality, we may assume that $\mathbf{c} \in \mathbb{Q}_{>0}^{2}$. 
3.2. There is a conjectural description of the two-sided cells in [1, Section 4.2] which we recall very briefly; more details can be found in both [loc.cit] and [11].

We assume $\mathbf{L}(s)=a, \mathbf{L}(t)=b$ with $a, b \in \mathbb{Q}_{>0}$ and set $d=b / a$. If $d \notin \mathbb{Z}$ then the partition is conjectured to be trivial, [1, Conjecture $\mathrm{A}(\mathrm{c})]$. If $d=r+1 \in \mathbb{Z}$ then let $\mathcal{P}_{r}(n)$ be the set of partitions of size $\frac{1}{2} r(r+1)+2 n$ with 2 -core $(r, r-1, \ldots, 1)$. A domino tableau $T$ on $\lambda \in \mathcal{P}_{r}(n)$ is a filling of the Young diagram of $\lambda$ with 0's in the 2-core and then $n$ dominoes in the remaining boxes, each labelled by a distinct integer between 1 and $n$ which are weakly increasing both vertically and horizontally. There is a process called moving through an open cycle which leads to an equivalence relation on the set of domino tableaux. This in turn leads to an equivalence relation on partitions in $\mathcal{P}_{r}(n)$ where $\lambda$ and $\mu$ are related if there is a sequence of partitions $\lambda=\lambda_{0}, \lambda_{1}, \ldots, \lambda_{s-1}, \lambda_{s}=\mu$ such that for each $1 \leq i \leq s, \lambda_{i-1}$ and $\lambda_{i}$ are the underlying shapes of some domino tableaux related by moving through an open cycle. The equivalence classes of this relation are called $r$-cells. [1, Conjecture $\mathrm{D}]$ conjectures that the two-sided cells are in natural bijection with the $r$-cells.

3.3. The result of this section is the following.

Theorem. Under the bijection (2) the $C M_{\mathbf{c}}$-partition of Irr $W$ is identified with the above conjectural description of the $K L_{\mathbf{L}}$-partition for $\mathbf{L}(s)=c_{s}, \mathbf{L}(t)=c_{t}$.

This theorem shows that core-quotient algorithm provides a natural identification of the $C M_{\mathbf{c}}$-partition and the conjectural $K L_{\mathbf{L}}$-partition. We do not know in general whether Lusztig's conjectured mapping from $\operatorname{Irr} W$ to the $K L_{\mathbf{L}}$-cells is given by this algorithm.

There are special cases where $[1$, Conjecture $\mathrm{D}]$ has been checked - for instance the asymptotic case $c_{t}>(n-1) c_{s}$, [1, Remark 1.3] - and thus in those cases we really do get a natural identification between the $C M_{\mathbf{c}}$-classes and $K L_{\mathbf{c}}$-cells.

3.4. We will need the following technical lemma to prove the theorem.

Lemma. Let $\lambda \in \mathcal{P}_{r}(n)$ and set $j=r$ modulo 2 with $j \in\{0,1\}$. Suppose that $s_{p q}$ is a $j$-removable box and $s_{t u}$ is a $j$-addable box such that $p \geq t$ and $q \leq u$ and there are no other $j$-addable or $j$-removable boxes, $s_{v w}$, with $p \geq v \geq t$ and $q \leq w \leq u$. Then there is a domino tableau $T$ of shape $\lambda$ and an open cycle $c$ of $T$ such that the shape of the domino obtained by moving through $c$ is obtained by replacing $s_{p q}$ with $s_{t u}$.

Proof. We use the notation of [11, Sections 2.1 and 2.3] freely. We consider the rim ribbon which begins at $s_{p q}$ and ends at $s_{t, u-1}$. We claim that this rim ribbon can be paved by dominoes. In fact this is a general property of a ribbon connecting a box, $s$, of residue $j$ and a box, $e$, of residue $j+1$. Let $R$ be such a ribbon. If $R$ contains only two boxes then $R=\{s, e\}$ so it is clear. In general the box adjacent to $s$, say $s_{\text {ad }}$, has residue $j+1$ so that $R \backslash\left\{s, s_{\text {ad }}\right\}$ is a ribbon of smaller length and so the result follows by induction. In our situation we can specify more. Starting at $s_{p q}$ we tile our rim ribbon, $R$, as far as possible with vertical dominoes up to and including $D=\left\{s_{p-m+1, q}, s_{p-m, q}\right\}$ where $s_{p-m, q}$ has residue $j+1$. If $s_{p-m, q}=s_{t, u-1}$ we have finished our tiling. Otherwise $s_{p-m, q+1} \in R$ so the square $s_{p-m, q+1}$ will be $j$-removable unless $\left\{s_{p-m, q+1}, s_{p-m, q+2}\right\} \subseteq R$. We now tile with as many horizontal dominoes as possible until we get to $E=\left\{s_{p-m, q+k-1}, s_{p-m, q+k}\right\}$ with $s_{p-m, q+k}$ 
having residue $j+1$. If $s_{p-m, q+k}=s_{t, u-1}$ then our tiling stops. Otherwise we must have the next domino as $F=\left\{s_{p-m-1, q+k}, s_{p-m-2, q+k}\right\}$ to avoid $s_{p-m, q+k+1}$ being $j$-addable. We can now repeat this process to obtain our tiling of $R$. From this description we obtain the following consequences. Let $s_{v w} \in R$ have residue $j+1$ and suppose that $s_{v w} \neq s_{t, u-1}$. Then

(i) The domino which contains $s_{v w}$ is of the form $\left\{s_{v+1, w}, s_{v w}\right\}$ or $\left\{s_{v, w-1}, s_{v w}\right\}$;

(ii) If $s_{v-1, w+1} \in \lambda$ then $s_{v, w+1} \in R$. Furthermore, if $s_{v w}$ is contained in a horizontal domino then $s_{v-1, w+1} \notin R$;

(iii) If $s_{v-1, w+1} \notin \lambda$ then $s_{v-1, w} \in R$.

Let $R$ denote the rim ribbon above and suppose it can be tiled by $t$ dominoes. Let $\mu$ be the shape $\lambda \backslash R$. In particular $\mu$ contains $\frac{1}{2} r(r+1)+2(n-t)$ squares. By the previous paragraph and [8, Lemma 2.7.13], $\mu$ is a Young diagram with the same 2-core as $\lambda$ and so there exists a $T^{\prime} \in \mathcal{P}_{r}(n-t)$ with shape $\mu$. Take such a $T^{\prime}$ filled with the numbers 1 to $n-t$. Now add $R$ to $T^{\prime}$. We can tile $R$ by dominoes by the previous paragraph and we fill the dominoes with the numbers $n-t+1, \ldots, n$ where the filling is weakly increasing on the rows and columns of $R$. This gives a domino tableau $T=T^{\prime} \cup R$ of shape $\lambda$.

We claim that $R \subseteq T$ is an open cycle, and that when we move through this cycle we remove $s_{p q}$ from $T$ and add $s_{t u}$. This will prove the lemma.

As we have seen in (i) a domino $D \subseteq R$ is either of the form $D=\left\{s_{v w}, s_{v+1, w}\right\}$ or $D=\left\{s_{v, w-1}, s_{v w}\right\}$ with $s_{v w}$ having residue $j+1$. In the case that $D=\left\{s_{v w}, s_{v+1, w}\right\}$ we have to study the square $s_{v-1, w+1}$ to calculate $D^{\prime}$. One of two things can happen. If this box does not belong to $T$ then $D^{\prime}=\left\{s_{v w}, s_{v-1, w}\right\}$ and $D^{\prime} \subseteq R$ by (iii). Otherwise $s_{v-1, w+1}$ does belong to $T$. In this situation the box is not in the rim so is filled with a lower value than $D$ and so $D^{\prime}=\left\{s_{v w}, s_{v, w+1}\right\}$. In particular, by (ii) above either $D^{\prime} \subseteq R$ or $D^{\prime}=\left\{s_{t, u-1}, s_{t u}\right\}$.

Now suppose $D=\left\{s_{v, w-1}, s_{v w}\right\}$. If the square $s_{v-1, w+1}$ is not in $T$ then $D^{\prime}=$ $\left\{s_{v-1, w}, s_{v w}\right\}$ and $D^{\prime} \subseteq R$ by (iii). If $s_{v-1, w+1}$ is in $T$ then it is not in the rim by (ii) and so is filled with a value lower than that of $D$. Thus $D^{\prime}=\left\{s_{v w}, s_{v, w+1}\right\}$ and $D^{\prime} \subseteq R$ unless $s_{v w}=s_{t, u-1}$, in which case $D^{\prime}=\left\{s_{t, u-1}, s_{t u}\right\}$.

It is now clear $R$ that is a cycle and moving through this cycle changes the shape of $\lambda$ by removing $s_{p q}$ and adding $s_{t u}$.

3.5. Proof of Theorem 3.3. We have $\boldsymbol{\theta}(\mathbf{c})=\left(-c_{s}+c_{t},-c_{t}\right)$ and by rescaling, see Remark 2.6(1), we consider $\boldsymbol{\theta}^{\prime}(\mathbf{c})=\left(1-\frac{c_{t}}{c_{s}}, \frac{c_{t}}{c_{s}}\right) \in \Theta_{1}$. The action of $\tilde{\mathfrak{S}}_{2}$ on $\Theta_{1}$ is given by $\sigma_{0} \cdot\left(\theta_{0}, \theta_{1}\right)=\left(-\theta_{0}, \theta_{1}+2 \theta_{0}\right)$ and $\sigma_{1} \cdot\left(\theta_{0}, \theta_{1}\right)=\left(\theta_{0}+2 \theta_{1},-\theta_{1}\right)$. The walls are $\left\{(d,-d+1) \in \Theta_{1}: d \in \mathbb{Z}\right\}$; they are of type $\{0\}$ if $d \in 2 \mathbb{Z}$ and of type $\{1\}$ if $d \in 1+2 \mathbb{Z}$. The fundamental alcove is $A_{0}=\{(d,-d+1): 0<d<1\}$, and the alcove $A_{r}=\{(d,-d+1): r<d<r+1\}$ is then labelled by either $\left(\left(\frac{r}{2}, \frac{-r}{2}\right), e\right) \in \mathbb{Z}_{0}^{2} \times \mathfrak{S}_{2}$ or $\left(\left(\frac{-r+1}{2}, \frac{r-1}{2}\right), \sigma_{1}\right) \in \mathbb{Z}_{0}^{2} \times \mathfrak{S}_{2}$ depending on whether $r$ is even or odd.

If $\frac{c_{t}}{c_{s}} \notin \mathbb{Z}$ then $\boldsymbol{\theta}^{\prime}(\mathbf{c})$ has type $\emptyset$ and the $C M_{\mathbf{c}}$-partition of Irr $W$ is trivial by Theorem 2.5 since $\emptyset$-classes are all singletons: this agrees with the conjectured triviality of the two-sided cells in this case. Thus we may assume that $r=\frac{c_{t}}{c_{s}}-1 \in \mathbb{Z}_{\geq 0}$. Then $\boldsymbol{\theta}^{\prime}(\mathbf{c})=(-r, r+1)$ will be in the closure of two alcoves, $A_{-r-1}$ and $A_{-r}$. We consider the latter. Let $\mathbf{s}$ be the element in $\mathbb{Z}_{0}^{2}$ coming from the labelling of $A_{-r}$; then $\tau_{\mathbf{s}}$ of (2) produces a bijection between $\operatorname{Irr} W=\mathcal{P}(2, n)$ and $\mathcal{P}_{r}(n)$. If we set $j=r$ modulo 
2 and $J=\{j\}$, the content of the theorem is simply the assertion that the $r$-cells in $\mathcal{P}_{r}(n)$ consist of the partitions in $\mathcal{P}_{r}(n)$ with the same $J$-heart.

Let us show first that if $\lambda, \mu \in \mathcal{P}_{r}(n)$ have the same $J$-heart, say $\rho$, then they belong to the same $r$-cell. The $J$-heart has no $j$-removable boxes, but we can construct the partition $\mu$ from $\rho$ by adding, say, $t j$-addable boxes. Now let $\nu$ be the partition obtained from $\rho$ by adding $t j$-addable boxes as far left as possible. We note that by [8, Theorem 2.7.41] $\nu \in \mathcal{P}_{r}(n)$. Of course $\mu$ and $\nu$ could be the same, but usually they will be different. Now we apply Lemma 3.4 again and again to $\nu$, taking first the rightmost $j$-removable box from $\nu$ to the position of the rightmost $j$-removable box on $\mu$ and then repeating with the next $j$-removable box on the successor of $\nu$. We continue until we have obtained a partition with shape $\mu$. By Lemma 3.4, this process is obtained by moving through open cycles. On the other hand, we can perform this operation in the opposite direction to move from $\lambda$ to $\nu$ via open cycles (for this we use the same algorithm and the fact that for a cycle $c$, moving through $c$ twice takes us back where we started, [4, Proof of Proposition 1.5.31]). It follows that $\lambda$ and $\mu$ belong to the same $r$-cell.

Finally, we need to see that if $\lambda, \mu \in \mathcal{P}_{r}(n)$ belong to the same $r$-cell, then they have the same $J$-heart. For this it is enough to assume that $\mu$ is the shape of a tableau obtained by moving through an open cycle on a tableau of shape $\lambda$. But in this case the underlying shapes differ only in some $j$-removable boxes, [11, Section 2.3], and so they necessarily have the same $J$-heart.

\section{Acknowledgements}

The second author gratefully acknowledges the support of The Leverhulme Trust through a Study Abroad Studentship (SAS/2005/0125).

\section{References}

[1] C. Bonnafé, M. Geck, L. Iancu, and T. Lam, On domino insertion and Kazhdan-Lusztig cells in type $B_{n}$, Progress in Math. (Lusztig Birthday Volume). To appear.

[2] R. Dipper and A. Mathas, Morita equivalences of Ariki-Koike algebras, Math. Zeit. 240 (2002), no. $3,579-610$

[3] P. Etingof and V. Ginzburg, Symplectic reflection algebras, Calogero-Moser space, and deformed Harish-Chandra homomorphism, Invent. Math 147 (2002), no. 2, 243-348.

[4] D. Garfinkle, On the classification of primitive ideals for complex classical Lie algebras. I, Compositio Math. 75 (1990), no. 2, 135-169.

[5] V. Ginzburg, N. Guay, E. Opdam, and R. Rouquier, On the category $\mathcal{O}$ for rational Cherednik algebras, Invent. Math. 154 (2003), no. 3, 617-651.

[6] I. Gordon, Baby Verma modules for rational Cherednik algebras, Bull. London Math. Soc. 35 (2003), no. 3, 321-336.

[7] — Quiver varieties, category $\mathcal{O}$ for rational Cherednik algebras, and Hecke algebras., Int. Math. Res. Papers (2008)

[8] G. James and A. Kerber, The representation theory of the symmetric group, Vol. 16 of Encyclopedia of Mathematics and its Applications, Addison-Wesley Publishing Co., Reading, Mass. (1981).

[9] G. Lusztig, Hecke algebras with unequal parameters, Vol. 18 of CRM Monograph Series, American Mathematical Society, Providence, RI (2003).

[10] M. Martino, The Calogero-Moser partition and Rouquier families for complex reflection groups (2008). ArXiv:0801.1627.

[11] T. Pietraho, Equivalence classes in the Weyl groups of type $B_{n}$, Journal of Algbraic Combinatrics 27 (2008), no. 2, 247-262. 
[12] R. Rouquier, Familles et blocs d'algèbres de Hecke, C. R. Acad. Sci. Paris Sér. I Math. 329 (1999), no. 12, 1037-1042.

School of Mathematics and Maxwell Institute for Mathematical Sciences, University of Edinburgh, James Clerk Maxwell Building, Kings Buildings, Mayfield Road, Edinburgh EH9 3JZ, SCOTLAND

E-mail address: igordon@ed.ac.uk

Mathematisches Institut, Universität Bonn, Beringstr. 1, D-53115 Bonn, Germany

E-mail address: mmartino@math.uni-bonn.de 